\title{
Historia de las glándulas paratiroides
}

\author{
Alfredo Jácome Roca*
}

${ }^{*} M D$, FACP. Internista-Endocrinólogo, miembro honorario de la Asociación Colombiana de Endocrinología, Diabetes y Metabolismo, miembro de número de la Academia Nacional de Medicina de Colombia y miembro activo de la Sociedad Colombiana de Historia de la Medicina.

Fecha de recepción: 5/05/2016

Fecha de aceptación: 30/06/2016

$\mathrm{E}$ ncontramos en la literatura médica numerosas y documentadas presentaciones monográficas sobre este tema ${ }^{(1-14)}$. A ellas hemos acudido, al igual que a otros artículos más detallados sobre aspectos específicos de esta interesante epopeya investigativa, a la que han contribuido también los estudios de casos memorables. Para no extendernos demasiado, hemos resuelto mencionar sólo marginalmente la historia de la vitamina D, o la del metabolismo óseo, calcitonina, osteoporosis y la de la osteodistrofia renal (hiperparatiroidismo secundario). Sobre la historia de la vitamina D contamos con excelentes revisiones que el lector interesado puede consultar $^{(15-19) .}$

Cuando tocamos temas históricos, generalmente nos referimos a los hechos y personajes que han participado en un descubrimiento, descripción o comprensión de algo hasta el momento desconocido. Sin embargo, en las ciencias de la vida es conveniente describir la historia evolutiva, que precede a cualquier descubrimiento biológico.

\section{Historia evolutiva}

El "mar primordial" en la Tierra fue originalmente rico en potasio y magnesio. Cuando aparecieron las células primitivas (con membranas y capacidad de autorreproducirse), este entorno marino fue cambiando muy lentamente, convirtiéndose en un medio rico en calcio y sodio pero pobre en magnesio y potasio. Posteriormente, al evolucionar estas células primitivas a una forma de vida más desarrollada, apareció un endoesqueleto calcificado ${ }^{(20)}$. Nuestros ancestros vertebrados fueron peces primitivos que vivieron en lagos y ríos durante el periodo siluriano y el ordovícico. Como la disponibilidad de calcio en estas aguas fue variable, estos organismos marinos empezaron a guardar depósitos de calcio. Dichos peces retornaron al mar y cambiaron el esqueleto osificado por cartílago, debido a la alta salinidad y concentración de calcio. Sin embargo, los peces mandibulados sí desarrollaron una glándula último branquial a partir de la cresta neural para producir calcitonina desde células $\mathrm{C}$ y así controlar una posible hipercalcemia.

Las glándulas paratiroides son de origen más reciente y aparecieron primero en los anfibios, a partir de la tercera y cuarta bolsas branquiales, pero sin originarse sus células en la cresta neural ${ }^{(21)}$. Las paratiroides aparecieron en seres vivos de nuestro planeta hace 360 millones de años -en la edad carbonífera- según algunos, aunque más probablemente lo hicieron hace 100 millones de años, según Greep ${ }^{(22)}$ en 1963, o según Pang y Epple ${ }^{(23)}$ en 1980. Como ya mencionamos, esto ocurrió en el contexto de la evolución de los anfibios y con la transición de organismos marinos rodeados de un ambiente rico en calcio a un medio terrestre o acuático que no lo era tanto. En aquellos que regresaron al entorno original, el citoesqueleto se cartilaginizó. En 1931, Denninger ${ }^{(24)}$ informó que en Illinois, Estados Unidos, ciertos esqueletos fosilizados mostraban evidencia de una osteotitis fibrosa quística (o enfermedad de Von Reklinghausen). Hallazgos similares habían sido informados en el antiguo Egipto.

\section{Paratiroides, tetania y osteítis fibrosa, antes del siglo XX}

Muchos siglos antes del descubrimiento de las paratiroides y de sus enfermedades, se habían observado las manifestaciones clínicas pero sin que se conociera su origen.

Inicialmente Courtial, un cirujano francés, describió en $1743^{(8)}$ los cambios óseos de la osteítis fibrosa quística en el paciente Pierre Siga, quien presentó compromiso óseo progresivo y murió a los 42 años; Siga tenía huesos tan blandos como la piel. Clark (1761-1815) informó "un tipo peculiar de convulsiones en niños" que ahora sabemos correspondían a una tetania. En Alemania, Steinheim (1789-1866) en 1830, y en Francia, Dance (1797-1838) describieron la tetania por hipoparatiroidismo un año más tarde ${ }^{(7)}$. El último reclamaba que se trataba de una enfermedad francesa (une paternité toute française".

En marzo de 1852, Sir Richard Owen (1804-1892), profesor y curador del Museo del Colegio Real de Cirujanos de Inglaterra, practicó en las instalaciones de su residencia una autopsia a un gran rinoceronte de la India (Rhinoceros unicornis) de un solo cuerno, anotando que el rinoceronte africano 
tiene dos cuernos. Este rinoceronte había sido adquirido 15 años antes por el zoológico de Londres, constituyéndose en una gran atracción. John Hunter mismo había comisionado al pintor Stubbs para que lo pintara, y este cuadro se conserva en el museo hunteriano del Colegio. Algunos han confundido a este rinoceronte macho con "Clara", famosísima rinoceronte hembra que recorrió Europa con presentaciones que enriquecieron a su dueño, y que murió en Londres cerca de un siglo antes.

Owen (figura 1) fue un famoso naturalista, paleontólogo y quien acuñó la palabra "dinosaurio"; fue colaborador de Charles Darwin, aunque luego mantuvo agrias controversias acerca de la selección natural y su teoría de la evolución; en la descripción de esta autopsia -y a pesar de que el cadáver del animal estaba muy descompuesto ya- describió en detalle un cuerpo glandular, compacto, pequeño y de color amarillo, anexo a la glándula tiroides donde emergen sus venas, siendo el primero en encontrar unas glándulas paratiroides, publicó su hallazgo en la revista Society's Transactions. Curiosamente ese año nacía en Suecia Sandström, quien habría de ser un experto en la morfología de las glándulas a las que denominaría "paratiroides".

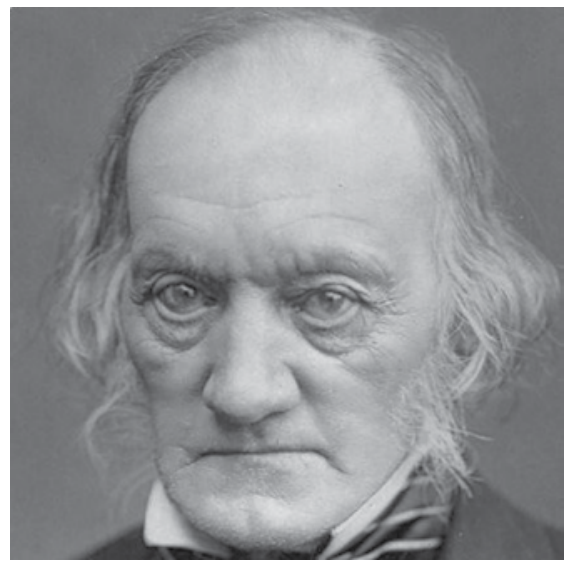

Figura 1. Sir Richard Owen

Un alumno suyo y profesor de anatomía las denominó “Glándulas de Owen", pero el epónimo no pegó. Ese mismo año, Corvisard ${ }^{(9)}$ introdujo el término "tetania" para cubrir el grupo de casos descritos hasta la fecha. Los signos de esta tetania hipocalcémica fueron descritos en su orden por Trousseau (1801-1865) en 1862, por Erb (1890-1921) y, finalmente, por Ghvostek (1835-1884) en 1876 $6^{(1,3,4)}$.

Armand Trousseau perteneció a aquel extraordinario grupo de clínicos franceses del siglo XIX, quien describiera otro "signo de Trousseau" (asociación de tromboflebitis con una neoplasia intraabdominal) y curiosamente, muriera con un cuadro clínico similar. Es nombrado también en la historia de la endocrinología porque adjudicó (o popularizó) epónimos como la enfermedad de Addison o la de Graves, entre otras ${ }^{(9)}$.

A esto debemos añadir información marginal recogida de otros autores, como el hecho de que Stanki en 1839 y Engel en 1864 hicieran descripciones óseas similares a las de Courtial, que Albers -en un atlas de anatomía publicado también en 1839- incluyera una ilustración de las paratiroides y que en Berlín, Robert Remak (1815-1865) describiera en 1855 las paratiroides del gato ${ }^{(1,3,4,9)}$. Este investigador fue el primer judío en ser nombrado profesor de una universidad prusiana y además asoció embriológicamente las paratiroides con el timo, en $1891^{(12)}$.

La existencia de las paratiroides en el hombre fue mencionada por el mismo Remak, además de Virchow en 1863. Estas glándulas fueron extensamente estudiadas algún tiempo después por un estudiante sueco de medicina, Ivar Victor Sandström (1852-1889) de Uppsala, quien señaló la existencia de dos paratiroides en cada lado del cuello en un meticuloso estudio anatomohistológico sobre 50 autopsias, encontrando en 43 cadáveres las formaciones que llamó glandulae parathyroidea por su situación cercana a la glándula tiroides ${ }^{(4,12,25,26)}$. Antes de identificar las glándulas en humanos, ya lo había hecho en perros, gatos, conejos, caballos y bueyes ${ }^{(26)}$. Algunos consideran infortunada esta denominación, pues se podría pensar que hacen parte de la tiroides o que tienen funciones similares a ésta.

Sandström procedía de una familia numerosa y de pocos recursos. Por esto demoró más años de lo normal cuando decidió estudiar medicina. En un verano en Upsala, logró conseguir un puesto como asistente temporal de investigación en el departamento de anatomía de la universidad local, bajo la dirección del profesor Edward Clason. Como anécdota marginal, el nuevo asistente hubo de acompañar a Clason y a otros dos profesores a la ejecución de un criminal, donde uno de ellos debía informar cuánto tiempo demoraba en perder la conciencia al observar la cabeza después de la decapitación. La ley decía que una vez muerto, el cadáver de un criminal pasaba a ser propiedad del Estado. Asistieron cerca de 2000 personas, incluso muchos niños. Podría calificarse de desastre pues pasaron al menos dos minutos antes de que finalizaran los movimientos y cambios observados en los ojos, mientras la gente corría a recoger la sangre del muerto, pues existía la creencia de que podía curar la epilepsia(7).

Este estudiante no sólo conocía de anatomía, sino también de histología, pues manejaba bien el microscopio y por años instruyó en su uso a otros estudiantes. Fue así como descubrió una glándula paratiroides, estudiando un material de tiroides canino. Observó una nueva estructura pero la consideró un tejido tiroideo en desarrollo, que se había detenido en su proceso embrionario. Sandström no conocía la descripción previa de Owen pero sí la mención sobre estas glándulas hechas por Remak y Virchow. Pero ellos no consideraron este tejido como independiente de la glándula tiroides. Su informe fue 
rechazado por editores alemanes, basándose en que era muy extenso (se trataba de una prestigiosa revista dirigida por Virchow, quien había mencionado con anterioridad la presencia de unas glándulas cercanas a la tiroides, del tamaño de un grano de arroz); además le restaba prestigio académico al trabajo el hecho de que su jefe, el profesor Clason, no figuraba como cautor $^{(25)}$.

El trabajo fue finalmente publicado en 1880 en una revista sueca local poco conocida (figura 2), lo que hizo que su descripción pasara desapercibida durante diez años, aunque posteriormente un resumen de él apareció en varias revistas alemanas, a raíz de lo cual Gley se enteró de estos hallazgos ${ }^{(27)}$. Algo similar le ocurrió a Paulesco en Rumania, quien habría descubierto la insulina antes de Banting y Best, pero publicó sus datos en una revista local desconocida.

Sandström sufría de melancolía; a raíz del embrollo generado por su publicación, lo despidieron del sitio donde trabajaba; además, su esposa e hijos lo abandonaron y finalmente se suicidó con un tiro de revólver, en casa de un hermano(7). Recordemos aquí el caso de Addison, otro melancólico a quien el rechazo por parte del British Medical Journal para la publicación de su histórico trabajo sobre la insuficiencia suprarrenal pudo influir en su decisión de suicidarse. Pero ambos quedarían en la historia por sus importantes descripciones primigenias.

Figura 2. Facsímil del artículo de Sandström

\section{UPSALA LÅKAREFÖRENINGS FÖRHANDLINGAR}

\section{Band. XV. \\ N:r $7 \& 8$}

SANDSTRõM, Glandulæ parathyreoideæ. - BLIX, Myograf. SANDSTröM, Prostatapreparat. - WIDE, Lefverpreparat. - HoLMGaEN, Retinaströmmen. - Svar till $H$. Cohn om färgblindheten. Föreningens yttrande mot $K$. Wickse/ls föredrag om dryckenskap.

\section{Om en ny körtel hos menniskan och atskilliga däggdjur \\ af}

Ivar SANDSTRöm.

För snart tre år sedan påträffade jag å sköldkörteln af en hund en liten, knappt hampfröstor bildning, som låg innesluten

\section{La conexión calcio}

Pareciera que el lento estudio de estos pequeños órganos estuviese por fin despegando, ya que en el mismo 1880, Anton Wiilfler (1850-1917), asistente de Virchow y luego profesor de cirugía en Graz y en Praga, se percató de unos tejidos presentes en el coloide tiroideo (probablemente paratiroides) y las llamó "restos fetales"(3). David Colley, en 1884, presentó en la sociedad londinense de patología la autopsia de una niña de 13 años que había muerto por la combinación de lesiones óseas sugestivas de hiperparatiroidismo (aunque Colley creyó que eran de osteomalacia) y litiasis del tracto urinario, siendo el primero en describir la asociación ${ }^{(4)}$.

En la década en que la información de Sandström permaneció en la oscuridad, se había vuelto popular la tiroidectomía total en Suiza para tratar el bocio endémico, común en ese país. Theodor Kocher (1841-1917) trabajaba en Berna cuando realizó numerosas tiroidectomías, al igual que Jacques-Louis Reverdin (1842-1929) en Ginebra; creían ambos que se trataba de una cirugía sin efectos adversos en el hombre. Reverdin publicó luego unos casos de anemia progresiva, con inercia física y mental posestrumectomía. Esto alarmó al bernés, quien se puso a ubicar a sus pacientes, consiguiendo revisar de nuevo a 34. De ellos, 20 presentaban grados variables de síntomas y signos compatibles con mixedema (Gull por aquella época ya había presentado los primeros casos de esta enfermedad). Al describir en 1883 el hipotiroidismo posquirúrgico, lo llamó “caquexia estrumipriva” y recomendó a otros cirujanos en un congreso que abandonaron la práctica de la tiroidectomía total, en favor de realizar la cirugía subtotal ${ }^{(29)}$. Los cirujanos se dieron cuenta además de que el posoperatorio con cualquiera de las dos técnicas (aunque más con la total) generaba cuadros de "tetania estrumipriva". Nathan Weiss (1851-1883) en 1881 estudió esta tetania en tres casos operados por Billroth (uno de los cuales falleció) pero consideró que no se trataba de una simple etapa de la enfermedad generada por la remoción de la glándula tiroides sino de algo diferente.

En esos mismos años, el famoso fisiólogo francés Eugene Gley (1857-1930) redescubrió en 1891 las paratiroides inferiores de conejo, y comenzó a investigar su fisiología y patología; Gley ${ }^{(27)}$ probaría que para que se produjera la tetania, las paratiroides debían ser extraídas junto con la tiroides y que eran esenciales para la vida; acudió a esa explicación (las paratiroides -por un tiempo llamadas glándulas de Gley- tendrían según él un efecto suplementario de la tiroides), cuando observó que la tiroidectomía no mata a los animales herbívo$\operatorname{ros}^{(24)}$. Gley -quien en su trabajo por fin citó a Sandström- confirmó datos anteriores del alemán Schiff, quien encontró que la tiroidectomía en gatos, perros y simios -pero no en conejosproducía una tetania que era fatal ${ }^{(1-3)}$. Pero Gley describió unos casos en los que la muerte no se produjo debido a la presencia de glándulas tiroides supernumerarias ¿Qué ocurría enton- 
ces? En los omnívoros -mas no en los herbívoros- las cuatro paratiroides estaban incrustadas dentro de la tiroides, y si bien se les adscribió inicialmente la misma función de la glándula de mayor tamaño, antes de terminar el siglo se confirmó que las glándulas más pequeñas tenían una función diferente. Finalmente, Kocher se dio cuenta (por las otras investigaciones y por su propia experiencia) que la (mal llamada) "tetania estrumipriva” se debía a la remoción inadvertida de las pequeñas glándulas paratiroides. Kocher, en 1889, informaría el encuentro de adenomas paratiroideos y en 1909 ganaría el Nobel de Medicina ${ }^{(28)}$.

\section{¿Tratan las paratiroides de compensar el proceso de osteítis fibrosa primaria?}

Vermeulen $^{(29)}$ pone en contexto las interpretaciones que se daban a los diversos hallazgos. Así, al celebrar los 70 años de Virchow en 1891, el patólogo alemán Friedrich Daniel von Recklinghausen (el mismo de la neurofibromatosis) hizo una contribución notable al informar tres casos de pacientes con tumores óseos -anteriormente considerados sarcomas- separando la osteítis fibrosa quística de otras enfermedades óseas como la osteomalacia y el Paget (figura 3); 50 años más tarde, Albright revisó los casos y encontró que dos tenían una "displasia fibrosa poliostótica”. Pero el tercer caso sí se trató de una verdadera osteítis fibrosa quística, y por esto el germano quedó con el epónimo a cuestas ${ }^{(29,30)}$.

El caso verdadero fue descrito detalladamente por Recklinhausen, y más adelante traducido al inglés por Albright. Se trataba del señor Bleich, un hombre de 40 años, que tuvo una serie de fracturas que se fueron presentando después de tratar la primera de ellas. Finalmente, el enfermo murió. Además

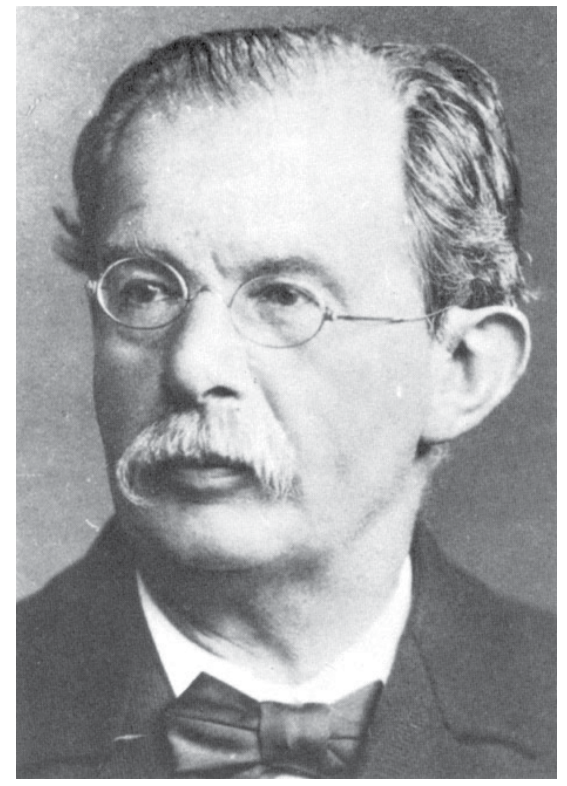

Figura 3. Friedrich Daniel von Recklinghausen del referido cuadro clínico, se practicaron detallados exámenes anatómicos y microscópicos del esqueleto, que resultaron compatibles con lo que después se conoció como un hiperparatiroidismo severo: deformidades esqueléticas múltiples, fracturas, fibrosis, pigmento hematógeno, quistes y conglomerados de células gigantes. De acuerdo con la nomenclatura de la época se hizo un diagnóstico de "osteosarcomas múltiples en una osteítis fibrosa", queriendo decir con la palabra "osteosarcoma" que se trataba de tumores pardos múltiples. En la autopsia, que Recklinhausen informó de manera manuscrita, encontró una glándula paratiroides aumentada de tamaño que no correlacionó con la patología ósea.

Esta falta de asociación no le ocurrió sólo al patólogo germano. Incluso Erdheim, en la primera década del siglo XX -al aportar nuevos datos sobre esta enfermedad- también estaba erróneamente convencido de que la lesión primaria estaba en el hueso y de que la hiperfunción de las paratiroides era secundaria además de que por esos días se pensaba que estas patologías óseas eran primarias, no secundarias a un tumor paratiroideo $^{(29)}$.

Entre 1895 y 1897 -Alfred Kohn (1867-1959) en Praga y Welsh, después- hicieron completas descripciones histológicas de las pequeñas glándulas. Kohn no creía en que se tratasen de variantes o etapas de desarrollo del tejido tiroideo. Al finalizar el siglo, De Sancti informó casos de adenomas paratiroideos, pero no los relacionó con la enfermedad ósea ${ }^{(12)}$.

\section{El siglo $\mathrm{XX}$}

Sabemos que el advenimiento de una nueva centuria no corta de tajo lo que venía ocurriendo en el siglo anterior. Todo ocurre lentamente, a pesar de la fecha exacta. A partir de los informes de Kohn, en 1900, Giulio Vassale (1862-1912), un patólogo de Modena, y Francesco Generali plantearon que la tetania era producida por una toxina, en consonancia con las ideas de la época. Estos mismos autores habían demostrado dos años antes que la remoción de las paratiroides del perro -dejando intacto el tiroides- producía convulsiones y tetania. Loeb produjo tetania en animales al provocar la precipitación del calcio, administrando oxalato de sodio, y Sabbatani hizo el mismo experimento pero logró corregir la tetania administrando sales cálcicas. En 1905, Max Askanasi describió y asoció los adenomas paratiroideos con la enfermedad esquelética observada en el hiperparatiroidismo primario ${ }^{(11,12)}$. Casos similares fueron informados por Schmorl (el de los nódulos) y por otros. Henry Dixon y colaboradores acuñaron el término "hiperparatiroidismo".

Como ya mencionamos, el patólogo austriaco judío Jacob Erdheim (1874-1937) hizo nuevos estudios sobre el tema que nos ocupa; describió en 1906 una hiperplasia de las paratiroides en pacientes con raquitismo y osteomalacia, sugiriendo en 1914 que la patología paratiroidea pudiera estar asociada con 
las lesiones óseas y que esto tenía que ver con el metabolismo del calcio; esto ya se trató de una nueva teoría. Acuñó el término "tetania paratiropriva", en remplazo de la tetania llamada estrumipriva por Kocher. Su aporte fue muy importante, pero su confusión sobre el origen de la lesión primaria nos deja ver que los conocimientos no se van adquiriendo en forma vertical, sino que el estudio posterior de los hallazgos va aclarando la fisiopatología.

La naturaleza endocrina de las paratiroides fue demostrada en 1909 por William G. MacCallum (1874-1944) y Carl Voegtlin (1879-1960) por medio de sus estudios de raquitismo renal, una situación patológica pediátrica en la que los niños desarrollan lesiones óseas secundarias a la insuficiencia renal $^{(31)}$. Estos autores observaron hipertrofia de las paratiroides y concluyeron que la reabsorción del calcio de los huesos se debía a que las paratiroides regulan el metabolismo del calcio. Su conclusión se vio reforzada cuando lograron, por medio de la administración de calcio, evitar la tetania que se presenta después de la extirpación de las paratiroides.

La actividad de las pequeñas glándulas (del tamaño de un grano de arroz) fue correlacionada con la osteítis fibrosa quística en 1915 por el patólogo vienés Federico Schlagenhaufer (1866-1930), quien observó que, que en estos casos, una sola paratiroides se encontraba aumentada de tamaño, sugiriendo que la causa de la enfermedad ósea era la hiperplasia adenomatosa paratiroidea y no al revés, como ocurre en los casos de osteodistrofia renal e hiperparatiroidismo secundario a falla del riñón ${ }^{(7)}$.

\section{La parathormona}

El francés G. Moussu en 1898 afirmó haber tratado satisfactoriamente a un paciente con tetania administrándole extracto acuoso de paratiroides equinas. Mantuvo una controversia con Gley acerca del papel que jugaba la tiroides versus la paratiroides en la tetania.

La parathormona fue aislada por Adolph A. Hanson (1880-1959) de Fauribault, MI, en 1913, y por James B. Collip (1892-1965), de Montreal, en 1915, (figura 4). Hanson ejercía en práctica privada, pero había sido alumno de Cushing, y en un garaje logró la experiencia, usando una técnica muy parecida a la del canadiense. Collip además demostró que su administración resulta en la disminución del calcio de los huesos e hipercalcemia. Hacia los años veinte aparecieron las primeras técnicas para medir el calcio, lo que facilitó la asociación de la calcemia con el nivel de hormona paratiroidea y el estudio de las diferentes patologías. El mismo Collip llamó parathormona (PTH) a un extracto muy activo de tejido paratiroideo en $1925^{(32)}$.

Este importante bioquímico -que como sabemos hizo parte muy activa del desarrollo de la insulina- logró resolver la controversia sobre la hormona paratiroidea como esencial

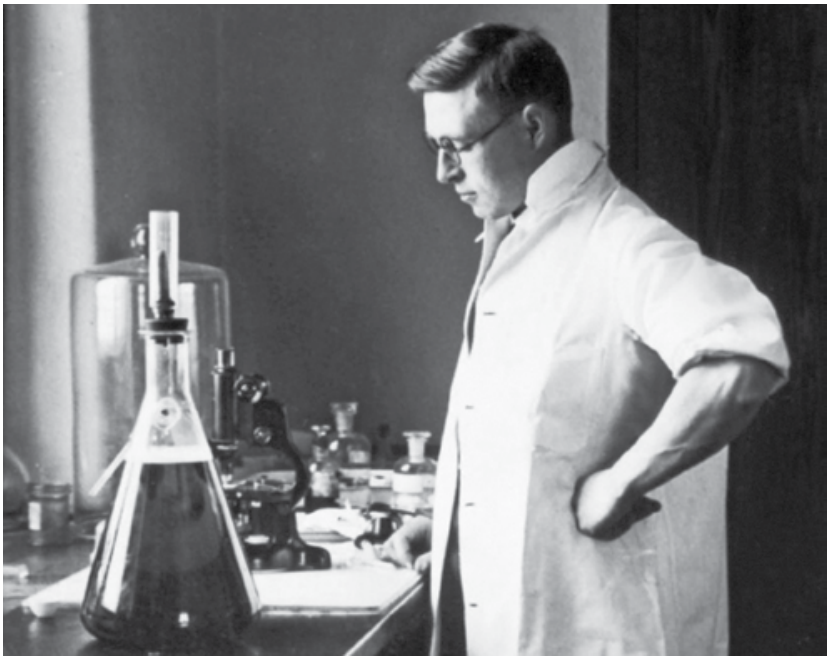

Figura 4. J.B. Collip en su laboratorio, 1927

reguladora de la calcemia, merced a una serie de experimentos. Preparó unos extractos de la glándula en ácido clorhídrico -para liberar la sustancia activa de otros tejidos como el estroma- y hacerla soluble. Mostró que estos extractos ácidos hacían desaparecer por completo la tetania paratiropriva y estableció que definitivamente las cuatro pequeñas glándulas eran endocrinas y segregaban PTH. Hanson reclamó la prioridad en los estudios con el ácido, pero su técnica tuvo dificultades cuando se trató de purificar y caracterizar el principio activo, asunto que quedó claro más de tres décadas después por el trabajo de Aurbach. La PTH fue purificada en 1959, más de cuatro décadas después de que Collip la aislara ${ }^{(8)}$.

El polipéptido hormonal obtenido por Collip resultó ser una serie de fracciones de la molécula, dado que el procedimiento de purificación rompía la secuencia en diferentes sitios, especialmente aquellos donde existía ácido aspártico o asparagina. En la década del 70, dos grupos de investigadores, Brewer y luego Sauer, lograron determinar la secuencia de la fracción amino terminal 1-34 en bovinos y en humanos, estableciendo que esa región era la fracción biológicamente activa de la hormona. Esta región 1-34 fue en los años setenta sintetizada por Potts y por Tregear, resultando en el desarrollo de radioinmunoanálisis ${ }^{(33)}$.

Koltz, en 1933, usó el AT-10 (dihidrotaquisterol) para tratar la tetania y nuevamente Collip -en 1934- mostró el efecto histológico directo de los extractos paratiroideos sobre los huesos en los animales nefrectomizados. Patt en 1942 demostraría que los niveles de calcio regulan la secreción de PTH. Windaus (quien ganó el Nobel de Química) investigó la estructura de las vitaminas D2 y D3 ${ }^{(16)}$.

\section{La cirugía de paratiroides}

En el siglo XIX, para el estudio de la tetania en animales se intentaron los autotrasplantes. En 1892, von Eiselsberg 
realizó un buen número de estos procedimientos, colocando las glándulas en el peritoneo de gatos, demostrando la desaparición de la tetania, la neoformación de vasos alrededor del tejido trasplantado y la reaparición de la tetania cuando se removían los trasplantes. Halstead, en el John Hopkins de Baltimore intentó el isoautotrasplante en perros, en 1909(34). Años más tarde, el trasplante de glándulas paratiroides, con el uso de diferentes técnicas, se usó mucho para el manejo del hipoparatiroidismo.

Oscar Hirsch intentó una paratiroidectomía en 1925, pero no tuvo éxito porque el paciente tenía una displasia fibrosa, no una osteítis. Ese mismo año se realizó la primera paratiroidectomía para tratar un caso de hiperparatiroidismo primario con osteítis fibrosa quística; la hizo en Viena Félix Mandl -un discípulo de Erdheim- en un paciente de nombre Albert Gahne. Este señor era un conductor de bus que habiendo participado como soldado en la Primera Guerra Mundial, había sido retirado del ejército a causa de una tuberculosis. Posteriormente, empezó a tener dolores óseos y astenia que lo incapacitaron para trabajar. En 1923 se le encontró una osteítis fibrosa quística a los rayos $\mathrm{X}$. Como se creía en esa época que los adenomas paratiroideos eran compensatorios para una deficiencia, le dieron inicialmente un extracto de tiroides y después otro de paratiroides. Luego Mandl le hizo un trasplante de glándula, procedente de un hombre recién muerto en un accidente, pero el enfermo empeoró. Después de hacer la exploración de cuello y remover el adenoma, desapareció la hipercalciuria y los huesos se recalcificaron. Sin embargo, siete años después (1932) hubo una recurrencia de la enfermedad y el paciente murió de falla renal ${ }^{(35-40)}$.

En Norteamérica, DuBois -del Bellevue Hospital de Nueva York- empezó a estudiar el metabolismo del calcio del apreciado capitán de fragata Charles Martell, quien había disminuído siete pulgadas de estatura en pocos años (figura 5). Sus radiografías mostraban una enfermedad ósea generalizada no bien definida, presentaba hipercalcemia, hipofosfatemia e hipercalciuria. Ya se sabía que la PTH producía este mismo cuadro bioquímico, por lo que sospechó un trastorno de las paratiroides, y resolvió remitírselo a Joseph C. Aub (18901973) en el Massachusets General Hospital (MGH), de quien había sido su maestro. Aub había utilizado la PTH de Collip para movilizar plomo de los huesos en un grupo de pacientes con intoxicación plúmbica y había confirmado los hallazgos del otro investigador acerca de su acción sobre la elevación de la calcemia (aumento de la calcemia y disminución de la fosfaturia). La hipercalcemia de Martell se confirmó y también la presencia de un balance de calcio negativo ${ }^{(35)}$.

Con un diagnóstico de probable hiperparatiroidismo primario fue sometido, en 1926, a una exploración de cuello por Edward Richardson en un lado, y un mes después del otro lado, y en estos procedimientos se extrajeron sendas paratiroides normales, sin lograr cambios en sus parámetros bioquímicos. Nuevamente al paciente se le practicaron -una y otra vez- in-
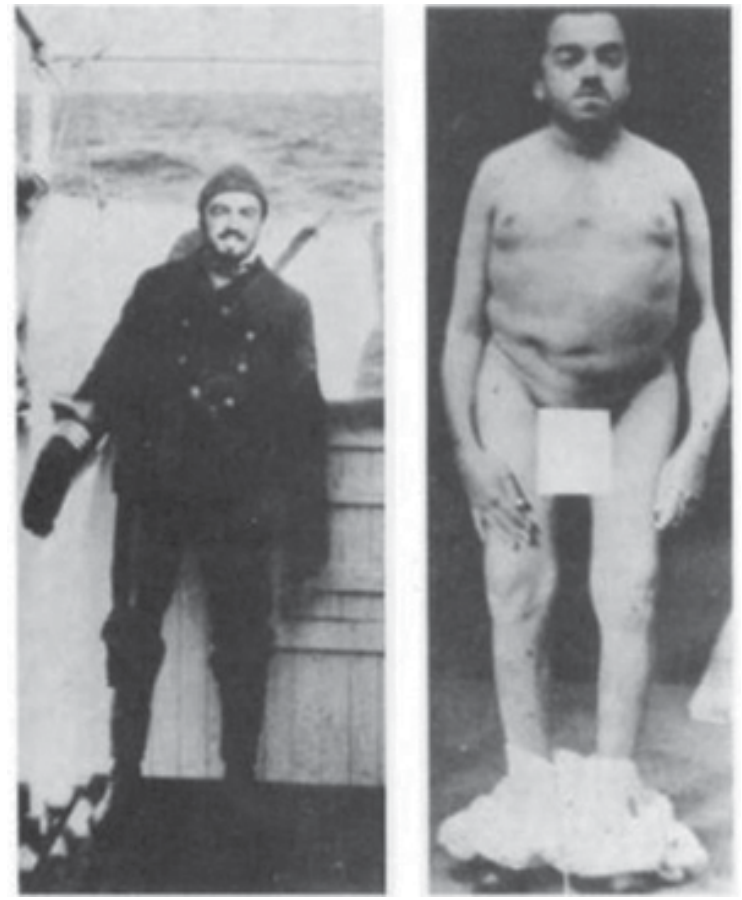

Figura 5. El capitán Martell, antes y después de su enfermedad

tervenciones para remover las otras glándulas paratiroides, pero su búsqueda resultó infructuosa. Como la primera cirugía ocurrió en 1926, se le ha designado como la primera paratiroidectomía en América. Se le indicó una dieta rica en calcio, con el fin de recalcificar sus huesos, lo que se logró parcialmente, al igual que tornar positivo el balance del calcio ${ }^{(39)}$. Pero la hipercalcemia empeoró, la calciuria aumentó y empezaron a presentarse cálculos a repetición en la orina. Llegó entonces la noticia de Europa sobre un paciente que había muerto por enfermedad de von Recklinghausen y a quien en la autopsia se le encontró un adenoma en el mediastino.

\section{Los "huesos hambrientos"}

Entonces Bauer ${ }^{(40)}$ reingresó al paciente y se le programó un esquema para lograr localizar el adenoma, lo que se logró en el séptimo intento (en 1932); el adenoma paratiroideo se hallaba en el mediastino anterior (por encima y al lado de la vena cava superior) y la extirpación normalizó la calcemia, pero el paciente murió, pues ya presentaba lesiones renales avanzadas y no le fue posible sobrevivir a la tetania (que se esperaba que ocurriera por el fenómeno denominado "huesos hambrientos", alteración debida a que los huesos están ávidos de calcio por la descalcificación asociada a la enfermedad).

Esta última operación fue realizada después de otras que habían curado a muchos pacientes con hiperparatiroidismo primario. Cope realizó tres de estas exploraciones, mientras que la final fue hecha por Edward D. Churchill, con Oliver Cope 
como ayudante. En un clásico artículo sobre la historia del hiperparatiroidismo en el MGH, Cope afirmó: “Este fue un final trágico para quien había contribuido tanto al conocimiento de la enfermedad, por su coraje, su comprensión y su demanda de que continuáramos adelante hasta que tuviéramos éxito"(39).

Vale la pena anotar aquí una anécdota. Cope disecó cadáveres para tener alguna experiencia previa a las operaciones de Martell; era experto en tiroidectomías pero nunca había reconocido paratiroides en un acto quirúrgico. Así que llamó a Albright para que estuviera presente en la cirugía y le mostrara las glándulas. Pero el endocrinólogo tampoco había visto alguna en un ser vivo.

Como anotamos anteriormente, desde comienzos del siglo XX los cirujanos notaron la mortalidad causada por severa hipocalcemia posttiroidectomía (y también posparatiroidectomía). Los miembros del Departamento de Cirugía del MGH hicieron numerosas exploraciones de cuello y resecaron paratiroides adenomatosas, por lo que pudieron describir en detalle la técnica quirúrgica. Utilizaron las biopsias por congelación y pensaron que para evitar la hipocalcemia era mejor realizar una adenomectomía parcial. Pero el tratamiento de esta complicación había mejorado. Los cirujanos la trataban con dietas ricas en calcio y bajas en fósforo, y calcio endovenoso. La vitamina D aún no estaba en uso.

\section{Estudios sobre el calcio}

No hay duda de que el progreso que introdujeron los estudios bioquímicos en la posibilidad de determinar clínicamente los niveles de calcio y fósforo, y en determinar los diferentes estados en que el calcio se encuentra en la sangre (ionizado, ligado a la albúmina y ligado a sales del tipo oxalato, citrato, fosfato, no reabsorbibles por el riñón), llevaron a numerosos estudios fisiológicos donde se demostraron los efectos del calcio ionizado sobre la contracción muscular, efectos sobre el corazón, secreciones, coagulación, etc. Es decir, la explicación de fenómenos como la tetania, la hipercalcemia, el papel regulador del riñón y todos los demás efectos que van más allá del simple efecto de sostén, al depositarse en los huesos, donde se encuentra el mayor reservorio de calcio en el organismo (1000 gramos de los 1200 que tiene un adulto). El calcio intracelular, el que está presente en la sangre, el del hueso y en los riñones, se encuentra en intercambio y constituye un importantísimo factor en el funcionamiento vital.

\section{El padre de la paratiroidología}

Fuller Albright (1900-1969) fue hijo de un financiero filántropo de Buffalo, creció en su hogar en Nueva Inglaterra y estudió medicina en Harvard donde se graduó de médico en 1924 (figura 6). Con un entrenamiento formal que envidiaría cualquiera en estos días, se especializó en medicina interna en el Johns Hopkins Hospital y luego estudió en Viena,

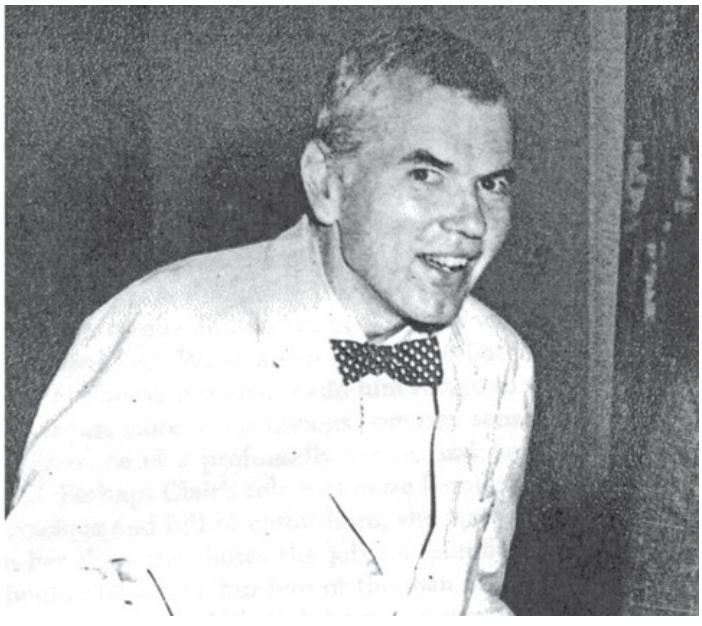

Figura 6. Fuller Albright

donde influenciaron su entrenamiento personas como Aub y Erdheim (quien había estudiado algún caso de carcinoma suprarrenal antes de Cushing, pero fue precisamente Albright quien le puso este último epónimo). También fueron importantes para él en aquella época Warfield Longcope y Ellsworth (en Baltimore), Hermann Zondek (en Berlín), J. Howard Means y Walter Bauer (en Boston). Albright volvió al MGH, donde desarrolló su fabulosa carrera que iluminó para siempre el campo del metabolismo óseo y de las hormonas calciotrópicas, y fue profesor en Harvard donde -con mentalidad de investigador puro- no quiso aceptar el cargo de profesor titular con tal de no tener tareas administrativas ${ }^{(35-40)}$. Aunque Albright es el padre del metabolismo del calcio y del fósforo (ya en 1929 postuló la teoría renal de la acción fosfatúrica de la PTH), la investigación que llevó a cabo en su famoso laboratorio biológico no fue exclusiva de este campo: participó en el uso inicial de los corticosteroides y llamó la atención sobre los peligrosos efectos colaterales de estas drogas (su síndrome favorito fue el de Cushing, contribuyendo a su conocimiento con muchos estudios); trabajó en litiasis renal y en los trastornos gonadales y de las hormonas sexuales, tanto que entre sus discípulos estuvieron Edward Conrad Reifenstein y Harry Fitch Klinefelter, además de otros muy nombrados como Hirsch Sulkowitch, Cockrill, Frederic Bartter, Russel Fraser, W. Parson y Charles Burnett. Albright realizó 118 publicaciones indexadas y describió tantos síndromes nuevos que se dio el lujo de ceder epónimos a personas como Reifenstein y Klinefelter (cuando alguien decía que había visto un caso de síndrome de Albright, inmediatamente se le contestaba: ¿cuál de todos? Al introducir un método muy bueno para medir la FSH, Albright pudo distinguir entre la disgenesia gonadal y otras formas de amenorrea primaria, y separar el hipogonadismo hipergonadotrópico con hialinización tubular testicular de otras formas de eunucoidismo. Como investigador tenía una facilidad innata para encontrar la respuesta a preguntas com- 
plejas, basado en los experimentos que observaba en la madre naturaleza, y siempre estaba desarrollando teorías para explicar la fisiopatología. Nunca hizo experimentos en animales sino que todos sus estudios fueron en seres humanos. Su investigación fue tan básica como aplicada que con él fue difícil fijar un límite. Muchos le reconocen la elaboración de conocimientos nuevos acerca de la osteoporosis, por lo que él le decía jocosamente a sus estudiantes que sobre este tema les había enseñado más de lo que él mismo sabía. Su ojo clínico podría resumirse en su idea de que un paciente que se queja de impotencia y pérdida de la libido no es un eunuco, pues estos últimos de eso no se quejan sino de que los confundan con una muchacha cuando hablan por teléfono. Escribió un conocido texto sobre paratiroidología, que fue referencia obligada sobre el tema ${ }^{(35-40)}$.

Sobre él dice lo siguiente el endocrinólogo argentino Carlos Mautalen, quien tuvo la oportunidad de realizar un entrenamiento con su grupo:

Su capacidad de trabajo, aunada a su claridad mental, una búsqueda libre de la verdad científica sin amarres a los dogmas vigentes, llevaron a Albright a describir un gran número de entidades clínicas nuevas y a la descripción de las secuencias fisiopatológicas que explicaban enfermedades ya conocidas. Entre 1935 y 1945 describió patologías como el hipoparatiroidismo idiopático y la posibilidad de su tratamiento mediante el At10, análogo de la vitamina $D$, la descripción y el tratamiento de la osteomalacia causada por la esteatorrea, la osteítis fibrosa renal, el raquitismo resistente a la vitamina $D$, la displasia fibrosa poliostótica (denominada también el síndrome de McCune Albright), la nefrocalcinosis con raquitismo y enanismo y su contraparte en el adulto, la acidosis tubular renal con osteomalacia, el pseudohipoparatiroismo (que por primera vez introdujo en la endocrinología el concepto de resistencia del órgano efector), y -diez años después- el seudoseudohipoparatiroidismo, los aspectos clínicos y fisiológicos del hiperparatiroidismo y la osteomalacia, introdujo por primera vez en endocrinología el concepto de resistencia del órgano efector, impulsó las mediciones del calcio y los estudios óseos, describió por primera vez el síndrome de Klinefelter, desarrolló el primer bioensayo para medir la FSH y profundizó los conocimientos de la enfermedad de Cushing ${ }^{(38)}$.

En la leída página web de datos biográficos Who named it? (www.whonamedit.com) de Ole Daniel Enersen, los siguientes epónimos se asocian con el profesor Albright (41):

- Anemia de Albright (asociada con hiperparatiroidismo avanzado).

- Profecía: en 1945 propuso el término "planificación familiar por tratamiento hormonal".

- Síndrome de Albright II. Sirve de ejemplo para demostrar cómo un síndrome en el que se observa una deficiencia hormonal, esta no existe realmente, sino más bien se trata de una incapacidad de respuesta por parte del órgano efector (tal como ocurre en el Klinefelter, en el hiperpara- tiroidismo secundario de la falla renal o el pseudohipoparatiroidismo).

- Prueba de Albright. Una prueba de tolerancia ácida sostenida por parte de los riñones, que valoran su capacidad para mantener el equilibrio ácido básico a través de la reabsorción.

- Enfermedad de Albright-Butler-Bloomberg. un síndrome metabólico caracterizado por enanismo marcado y otras anormalidades severas del desarrollo.

- Síndrome de Albright-Hadorn. Parálisis muscular hipocalémica paroxística asociada con osteomalacia en pacientes con acidosis tubular renal, epónimo que ahora se considera obsoleto, al igual que el síndrome de Lightwood-Albright, una forma de acidosis tubular renal que aparece en el primer año de vida.

- Síndrome de Forbes-Albright. Galactorrea-amenorrea asociada con macroprolactinoma. Este término, al igual que otros relacionados como el de Chiari-Frommel (descrito en el siglo XIX) y el de Ahumada-Del Castillo, han entrado en obsolescencia con los nuevos métodos diagnósticos, ya que en la época en que fueron descritos no se conocía la prolactina ni la presencia de microprolactinomas.

- Síndrome de McCune-Albright. Una anomalía congénita marcada por displasia fibrosa poliostótica que se asocia con manchas café con leche -como en la neurofibromatosis-y trastornos endocrinos tipo pubertad precoz.

- Síndrome de Martin-Albright. Caracterizado por corta estatura, cara redonda, cuello grueso y extremidades cortas en relación con el tronco (Si Albright viviera actualmente, hubiese sido genetista). Podría tratarse del llamado fenotipo Albright (AHO) consistente en braquidactilia, cara redonda, talla baja, obesidad, osificaciones subcutáneas y retardo mental, que se ve en ciertos tipos de pseudohipoparatiroidismo.

- Síndrome de Morgagni-Turner-Albright. 0 síndrome de Turner, endocrinopatía congénita caracterizada por corta estatura, características sexuales secundarias ausentes, cuello palmeado, cardiopatías congénitas y cariotipo XO, una forma de disgenesia gonadal.

- Síndrome de Klinefelter-Reifeintein-Albright. Hipogonadismo hipogonadotrópico asociado a hialinización tubular testicular, retardo mental, fenotipo característico y cariotipo XXY.

Según su colaborador A.P. Forbes, hace más de medio siglo que Albright hizo fundamentales aportes al conocimiento de la osteoporosis, que pueden resumirse en estos seis puntos:

1. Reconoció que existía una deficiencia en la formación ósea, no en la mineralización de la matriz ósea.

2. Observó que cuarenta de cada cuarenta y dos pacientes con osteoporosis antes de los 65 años eran mujeres posmenopáusicas y jóvenes ooforectomizadas.

3. Concluyó que los estrógenos estimulan los osteoblastos (teoría después refutada, ya que su acción es antirresortiva). 
4. Demostró por estudios de balance metabólico que los estrógenos causan un balance positivo de calcio en la osteoporosis posmenopáusica.

5. Introdujo la progesterona para administrarla periódicamente para tratar la hiperplasia endometrial que se origina de la prolongada terapia estrogénica.

6. Mostró que la terapia estrogénica a largo plazo detenía el daño vertebral y la pérdida de estatura que ocurre en esta osteoporosis menopáusica y prevenía su aparición temprana. Él se valía de radiografías convencionales para estudiar el hueso, no de las más sensibles densitometrías actualmente disponibles y de los grandes estudios clínicos que posteriormente indicaron huesos más fuertes y menos fracturas con los estrógenos.

Albright, que -como hemos dicho- es el perfecto enlace entre la endocrinología clásica y la molecular, fue una víctima prematura de la enfermedad de Parkinson, cuyos primeros síntomas aparecieron antes de que cumpliera los 40 años. Uno de sus comentarios sobre una enfermedad que hacía que hasta el más breve comentario requiriera un tremendo esfuerzo, fue aquel que decía que si la enfermedad de Parkinson hubiese estado dentro de su campo de interés investigativo, hubiera resuelto el problema hace tiempo. Esto nos recuerda otro autosuficiente comentario de similar corte, el de Oscar Wilde que decía: "cuando quiero leerme un buen libro, lo escribo". Su progresivo impedimento hasta llegar a la silla de ruedas y la dificultad para hablar (nos imaginamos que a la cara de máscara y a otras manifestaciones clásicas de este trastorno) no le impidió trasladarse a Suramérica para observar y estudiar pacientes con displasia fibrosa poliostótica que habían sido informados originalmente en dicha región. Quiso voluntariamente someterse a aquellas quimopalidectomías que desarrolló el neurocirujano Irving Cooper, a pesar de que este y su médico de cabecera se oponían. Le fue bien en la primera operación -con recuperación aceptable de las funciones del lado derecho- pero en la segunda, infortunadamente presentó una hemorragia cerebral masiva que le quitó el habla y luego le envió a un coma que duró trece años. Nos cuenta el endocrinólogo Efraim Otero Ruiz que -durante su tiempo en el MGHel eminente investigador yacía (seguramente conectado a tubos y demás instrumentos para preservar la vida) mientras se acostumbraba pasar por su cuarto para vivir la impactante experiencia de observar un muerto en vida. Fue prematura e incapacitante su enfermedad, aunque no tanto su cronología, ya que murió de 69 años.

A mediados del siglo pasado se había logrado una considerable experiencia quirúrgica en el tratamiento del hiperparatiroidismo. Algunas casuísticas de aquella época hablaban de 140 casos de la Mayo Clinic, 138 de Estocolmo, 230 del MGH y 95 de Irlanda del Norte, entre otros ${ }^{(35-40)}$.

Cope alcanzó un número impresionante de remoción de adenomas paratiroides, teniendo en cuenta la época. A medida que aumentó la experiencia quirúrgica, se vio que el adenoma único no era la única causa del hiperparatiroidismo primario; en su serie de 200 pacientes, Cope describió adenoma único en $79 \%$, adenoma doble en $5 \%$ e hiperplasia en $7 \%$ de los casos. En el MGH de Boston, uno de los endocrinólogos era Fuller Albright, los cirujanos de paratiroides eran Edward Churchill y Oliver Cope, mientras que el patólogo era Benjamín Castleman. Con Churchill, hizo Cope la primera publicación en 1934 sobre los primeros 11 casos de hiperparatiroidismo tratados con operación. En 1936 informó 30 casos y en 1941 planteó la ocurrencia de adenomas paratiroideos en el mediastino anterior, por lo sugirió la división de la exploración en dos etapas. Para 1942 llevaban ya 67 casos operados de adenomas paratiroideos en una década, cifra que actualmente superan en un solo mes cirujanos endocrinos de grandes centros universitarios de referencia, aunque -valga decirlo- la mayoría de los casos son hiperplasias secundarias a falla renal. Un año más tarde informó los primeros casos de hiperplasia generalizada y para 1953, los primeros cuatro casos de carcinoma de paratiroides, entre un total de 148 casos operados.

\section{Nuevos estudios}

En 1948, Barnicot mostró que el injerto de tejido paratiroideo adyacente al hueso tiene un efecto descalcificante local y directo y, tres años más tarde, Chang comprendió las relaciones entre el calcio, la PTH y la vitamina D. En 1962, Copp ${ }^{(42)}$ descubrió la calcitonina. Pearse -estudioso del sistema APUDacuñó el término de células $\mathrm{C}$ del tiroides, productoras de la anterior hormona. Luego Neer estudió la calciocinética con métodos radiactivos y Reiss midió PTH por radioinmunoanálisis; en 1970, Brewer informó la secuencia de aminoácidos de la PTH bovina mientras Boyle y Héctor DeLuca lograron la síntesis renal de la 1-alfa-25, dihidroxicolecalciferol, una nueva hormona, y en 1971 Aus demostró que la acción de la PTH se hace a través del segundo mensajero AMPc. Gerald Aurbach fue quien aisló la parathormona ${ }^{(19)}$.

A partir de la década de los sesenta, los estudios se concentran en la importancia de la nueva hormona calcitonina -actualmente colocada en un lugar secundario- en la investigación de la vitamina D como hormona, en la aparición de nuevos receptores, de síndromes paraneoplásicos (especie de laboratorios de la naturaleza) y de la aplicación de nuevas tecnologías diagnósticas. Aparecen el alendronato y sucedáneos como drogas estrella para la prevención y tratamiento de la osteoporosis (en combinación con las sales de calcio), las que se administran también en ciertos tumores óseos como el mieloma múltiple. El estudio exhaustivo del metabolismo cálcico en los casos de urolitiasis pierde importancia por no ser costoefectivo, por lo que este se deja para los casos recurrentes en los que se sospeche algún tipo de trastorno como hiperparatiroidismo o hipercalciuria idiopática. 


\section{Aportes en Colombia}

Los colombianos participamos con casuística sobre este tema. En 1957 apareció el primer número de la Revista de la Sociedad Colombiana de Endocrinología en el que se publicaron tres trabajos sobre estas patologías, que fueron glosados en el importante Yearbook of Clinical Endocrinology. En el mismo año en que Albright encontró que el hiperparatiroidismo se asocia con pancreatitis, en Colombia Ucrós y Gómez informaron tres pacientes con un posible pseudo-pseudo-hipoparatiroidismo, descrito por el investigador de Boston cinco años antes; además, Malagón y Cabrera describieron cinco pacientes con hipofosfatasia.

Los dos primeros pacientes con adenoma paratiroideo fueron vistos en el Hospital San Vicente de Paul en Medellín ${ }^{(43)}$. Años más tarde aparecieron más casos de estas patologías, estudiados en el Hospital San Juan de Dios -donde se iniciaron las primeras dosificaciones hormonales por radioinmunoanálisis- y en otros hospitales como el Militar, San Ignacio, Evaristo García y San Vicente de Paul. El cirujano Rafael Casas Morales -de la Universidad Nacional- fue el primero en coleccionar una importante casuística de hiperparatiroidismo primario (figura 7); admitido mediante beca de American Cyanamid en el Massachusetts General Hospital de la Universidad de Harvard como Fellow en Cirugía trabajó bajo la tutoría de William McDermott, Jr. y luego de Oliver Cope ${ }^{(44)}$. El primero dedicado a la hipertensión portal y el segundo a la cirugía endocrina, motivo por el cual el doctor Casas desarrolló gran interés en la cirugía de tiroides y paratiroides. En virtud de la amistad que desarrolló con Cope, consiguió traerlo a Bogotá por un par de semanas para organizar un servicio de cirugía endocrina en el Hospital San Juan de Dios. Casas logró coleccionar y analizar

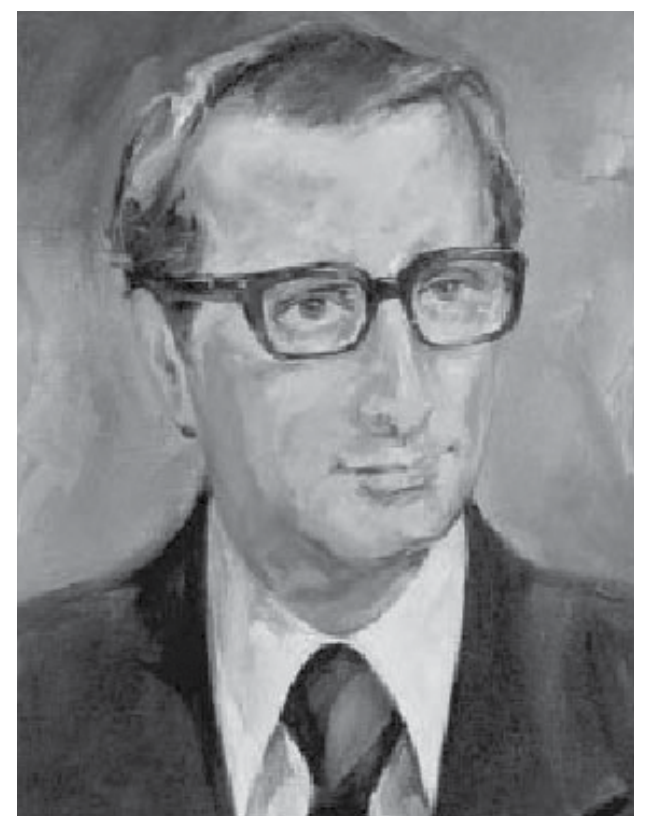

Figura 7. Rafael Casas Morales
25 casos de hiperparatiroidismo primario (la mayoría suyos, otros de varias instituciones del país), que fueron publicados años más tarde en un bello libro titulado Hiperparatiroidismo en Colombia, editado por el Instituto Nacional de Salud ${ }^{(44)}$. Este libro se habría quedado inédito si no hubiera sido por el endocrinólogo Enrique Ardila, quien encontró casualmente el manuscrito en una gaveta del escritorio del profesor.

Vendría la asociación de esta enfermedad con una pancreatitis aguda, forma como precisamente murió una paciente incluida en la casuística del profesor Casas Morales, correspondiéndole al autor de este artículo practicar la autopsia y presentar el caso como residente de patología en el tradicional Hospital San Juan de Dios de Bogotá. Una revisión retrospectiva de sus placas óseas con los radiólogos, mostró cambios compatibles con osteítis fibrosa quística (en la autopsia se observaron los brown tumors, pero lo radiólogos habían diagnosticado "encondromatosis múltiple" en su estudio de años anteriores).

Para esa misma época, el director del Instituto -Antonio Iglesias- publicó también dos gruesos volúmenes de su autoría principal con la colaboración de otros, presentando exhaustiva información sobre el metabolismo óseo ${ }^{(15)}$; Iglesias y colaboradores $^{(19)}$ escribimos la Historia de la vitamina D. Con ocasión del lanzamiento de la calcitonina en Colombia, el autor de estas notas escribió un opúsculo (figura 8) sobre el tema, que tuvo amplia circulación en países bolivarianos ${ }^{(13)}$. También se publicó un número sobre paratiroides en la Revista de la Sociedad Colombiana de Endocrinología.

En el Hospital Universitario San Ignacio estudiamos 8 casos (45-47), los 6 primeros presentaron dificultades en el diagnóstico diferencial ante posibilidades como diabetes insí-

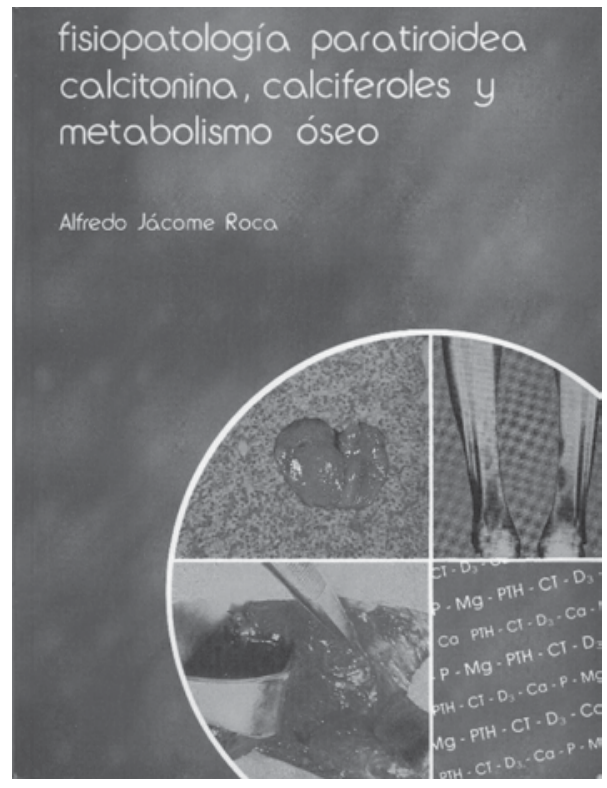

Figura 8. Libro Fisiopatología paratiroidea, calcitonina, calciferoles y metabolismo óseo 
pida, mieloma múltiple, úlcera péptica con dolor lumbar, carcinoma de tiroides con metástasis óseas y lesión traumática de hueso con antecedente de ureterolitotomía por cálculo renal ${ }^{(45)}$. El estudio de estos enfermos condujo a establecer una hipercalcemia de tipo primario, la mayoría con osteítis fibrosa quística, siendo de ayuda la prueba de supresión con cortisona practicada en tres pacientes; posteriormente condujo a la exploración y extirpación quirúrgica de las glándulas paratiroides, para lo cual fue orientador el esofagograma en la localización de los adenomas en tres oportunidades. En cada caso se extirpó un adenoma paratiroideo único: uno de células oxifílicas, otro de células claras y los restantes de células principales. Todos presentaron crisis tetánicas posquirúrgicas, pero luego hubo remisión de la enfermedad. A diferencia de los 6 anteriores $^{(46,47)}$ en donde el diagnóstico se basó más que todo en hallazgos radiológicos de lesión ósea y/o litiasis renal, hipercalcemia y métodos indirectos de localización del adenoma, en los dos últimos pacientes se utilizaron métodos modernos tales como dosificación de calcio por absorción atómica, PTH, TAC y ultrasonografía de paratiroides; en ninguno de estos dos casos se evidenció radiológicamente la presencia de lesiones óseas y ambos presentaron nefrolitiasis, aunque antes de esto a uno de ellos se le había encontrado una hipercalcemia asintomática en un chequeo rutinario.

Para los años 70, las series de casos más grandes pertenecían a la Clínica Mayo con 319 pacientes de 1970 a 1973, al MGH con 332 pacientes de 1962 a 1975, al Instituto Karolinska con 129 pacientes de 1961 a 1971, al Henry Ford Hospital con 121 pacientes de 1960 a 1974 y a la Universidad de Göteborg con 170 pacientes de 1970 a 1974. El 85\% de los casos tenían adenoma paratiroideo y de éstos la gran mayoría eran adenomas únicos; rara vez existen dos o tres tumores. Al 14\% de los pacientes se les ha encontrado hiperplasia de las cuatro paratiroides y a un $1 \%$ carcinoma $^{(45)}$.

Hay que resaltar los esfuerzos hechos por personas como Enrique Ardila Ardila, internista-endocrinólogo especialista en metabolismo óseo, quien -además de ser uno de los fundadores (con Iglesias-Gamarra y otros) de la Sociedad Colombiana de Osteología y Metabolismo y de su importante revista- es el editor (junto con Carlos Mautalen de Buenos Aires, exdiscípulo de Albright y Camilo Jiménez, de la Universidad de Texas, en Houston) del importante libro Osteoporosis en Iberoamérica, cuya segunda edición la publicó la editorial Manual Moderno, Bogotá 2012 (figura 9). Con la participación de 49 autores latinoamericanos, de Estados Unidos y España, el libro está dividido en 5 secciones, 36 capítulos y totaliza 585 páginas. A diferencia de los textos clásicos, dicho libro está dirigido a orientar al clínico con capítulos que abordan aspectos prácticos del estudio y el tratamiento de los pacientes con osteoporosis. Podemos decir que en esta opera magna, los mejores expertos iberoamericanos están presentando su experiencia con esta patología, que está absorbiendo casi todos los esfuer-

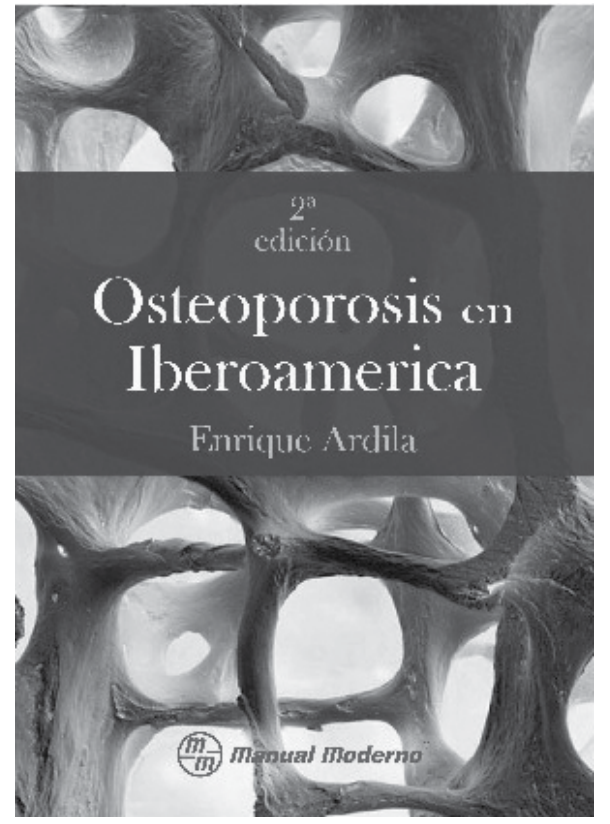

Figura 9. Segunda Edición del Libro Osteoporosis en Iberoamérica, de Enrique Ardila, Carlos Mautalen y Camilo Jiménez, Editorial Manual Moderno (Bogotá-México).

zos investigativos sobre metabolismo óseo por su frecuencia e impacto en la salud; esto se ha visto facilitado con la presencia de las técnicas de densitometría, mediciones de iones, de hormonas y de diferentes marcadores del metabolismo óseo ${ }^{(48)}$. Otro tema importante es el de la osteítis hiperparatiroidea de los renales crónicos, que afortunadamente puede controlarse con la administración de calcitriol.

Para finalizar este artículo debemos observar que de aquel cuadro clínico presente en un $2 \%$ de los casos actuales, con síntomas severos de dolor óseo, fracturas patológicas, marcadas anormalidades radiológicas debidas a los "tumores pardos", litiasis renal, síntomas digestivos inespecíficos de estreñimiento, náusea y vómito, dispepsia (probablemente relacionados con hipercalcemia, gastritis o pancreatitis), hemos pasado al frecuente caso del hiperparatiroidismo primario asintomático, muchas veces sin una verdadera indicación quirúrgica. Conocemos mejor la patología, las bases genéticas (como en la adenomatosis endocrina múltiple), tenemos mejores formas imaginológicas para detectar adenomas (como la ecografía con sestamibi) y disponemos de mejores técnicas quirúrgicas, incluyendo las laparoscópicas. El manejo ahora tiende a ser más conservador, particularmente en las personas mayores de 60 , con hipercalcemias y niveles de parathormona elevados en forma leve a moderada. $Y$ en los casos quirúrgicos se están imponiendo técnicas laparoscópicas, gracias también a una mejor tecnología imaginológica para localizar adenomas. 


\section{Referencias}

1. Gasparri G, Camandona M, Palestini N. History of parathyroids. In: Primary, Secondary and Tertiary Hyperparathyroidism: Diagnostic and therapeutic updates. Guido Gasparri, Michele Camandona,Nicola Palestini (Editors). Springer International Publishing AG, Part of Springer Science + Business Media. 2016; pp:1-7.

2. Organ CH. The History of Parathyroid Surgery, 1850-1996: The excelsior medical society 1998 Edward D. Churchill lecture. J Amer Coll Surg. 2000; 191: 191: 284-299.

3. Kafetzis ID, Diamantopoulos A, Christakis I, Leoutsakos B. The history of the parathyroid glands. Hormones 2011; 10 (1): 60-80.

4. Rowlands BC. Hyperparathyroidism: an early historical survey.Principio del formulario Springer ShopAnn R Coll Surg Engl. 1972; 51(2): 81-90. Amazon.com.

5. Dorairajan N, Pradeep PV. Vignette Hyperparathyroidism: Glimpse into Its History. Int Surg: 2014; 99 (5): 528-533.

6. Eknoyan G. A history of the parathyroid glands. Am J Kidney Dis. 1995;26(5):801-7.

7. Taylor S. Hyperparathyroidism: retrospect and prospect. Ann R Coll Surg Engl. 1976; 58(4): 255-265.

8. Amaro-Méndez S. Breve historia de la endocrinología. Editorial CientíficoTécnica, La Habana 1975.

9. Jácome Roca A. El metabolismo óseo. En: Historia de las Hormonas. Academia Nacional de Medicina (Bogotá). Pp 149-166.

10. Jácome A. Notas históricas sobre paratiroidología. Rev Soc Col Endocrinol 1982; 13:88-93.

11. Medvei VC. A History of Endocrinology. Lancaster, Lancashire, England. MTP Pr; 1982.

12. Nordenström J. Sandström's discovery, in: The Hunt for the Parathyroids 2012; John Wiley \& Sons, Oxford. doi: 10.1002/9781118343371.ch1

13. Jácome-Roca A. Fisiopatología paratiroidea, calcitonina, calciferoles y metabolismo óseo. 1985. Bogotá, Gráficas Esfera.

14. Vassallo M, Blanco DV. Revisión histórica de la fisiología de las glándulas paratiroides. Rev venez cir. 2011; 64(1):24-33.

15. Iglesias-Gamarra A, Vásquez-Lamadrid J. Enfermedades metabólicas del hueso, tomo II. 1992. Instituto Nacional de Salud, Bogotá.

16. Iglesias-Gamarra A, M Peña Cortés, J Félix Restrepo, F Rondón y Cols. Osteomalacia y raquitismo. Análisis y estudio en diferentes períodos históricos en Colombia. REEMO 2000;9:216-221.

17. Kodicek E. The story of vitamin D, from vitamin to hormone. Lancet 1974;1: 325-329.

18. DeLuca HF. Vitamin D endocrinology. Ann Intern Med 1976; 85. 366-377.

19. Iglesias A, Restrepo JF. Del raquitismo a la vitamina D. En: Historia de la vitamina D (Antonio Iglesias Gamarra, Editor). Ediciones Universidad Simón Bolívar, Barranquilla, 2008.

20. Rasmussen $\mathrm{H}$. The cellular basis os mammalian calcium homeostasis. Clin Endocrinol Metab, marzo 1972, pags.3-20

21. Copp DH. Evolution of calcium regulation in vertebrates. Clin Endocrinol Metab, marzo 1972, pags. 21-32.

22. Greep RO. Parathyroid hormone. In Comparative Endocrinology. Ed. Von Euler, V.S. Heller H. New York Academic Press; 1963; pp:325-370.

23. Pank RKT, Epple A. Evolution of the vertebrate endocrine systems. Graduate Studies Lubbock, Texas, 1980. Texas Tech University.

24. Denninger HS. Osteitis fibrosa in a skeleton of a prehistoric American Indian. Arch Path 1931; 11: 939-94.
25. Sandström I. Omen ny Kortel hos mennisken och atskilige baggdjur. Upsala Lakareförenings Förhandlingar band. 1880; 15:441-471.

26. Aidan CJ. The Glandulae Parathyroideae of Ivar Sandstrom: Contributions from Two Continents. Am J Surg Pathol. 1966; 20(9):1123-1144.

27. Gley E, 1891 Sur les fonctions du corps thyroide. C Soc Biol 43: 841-847.

28. The Nobel Prize Internet Archive. Winners of the Nobel Prize in Physiology and Medicine. www.nobelprizes.com

29. Vermeulen AHM. The birth of endocrine pathology. How Erdheim misunderstood parathyroids. Virchows Arch. 2010 Sep; 457(3): 283-290.

30. Von Recklinghausen FB. Die fibroese oder deform-inzende Ostitis die Osteomalakie und die Osteoplastis de Carcinose in ihren regenseitigen Beziehungen In festehrift. 1891. R. Virchow, Berlin.

31. MacCallum WG, Voegtlin C. On the relation of tetany to the parathyroid glands and to the calcium metabolism. J Exp Med 1909; 11: 118-15.

32. LibreriadelaU.comCollip JB: The Internal Secretion of the Parathyroid Glands. Proc Natl Acad Sci 1925; 11: 484-485.

33. Cano F, Jara A. La osteodistrofia renal y la parathormona supresora de la remodelación ósea. Rev chil pediat, 2006; 77(2): 127-137.

34. Halstead WS, 1909 Auto and isotransplantation in dogs of the parathyroid glands. J Exp Med 11: 175-199.

35. Albright F, Bloomberg E, Castleman B, Churchill E. Hyperparathyroidism due to diffuse hyperplasia of all parathyroid glands rather than adenoma of one. Arch Inter Med 1934. 54:315-329.

36. Albright F, Reifenstein FC. The parathyroid glands and metabolic bone disease. 1948.

37. Forbes AP. Fuller Albright, his concept of postmenopausal osteoporosis and what came of it. Clin Orthop Relat Res. 1991; 269:128-41

38. Mautalen C. Fuller Albright. Un tributo al padre de las osteopatías metabólicas a cien años de su nacimiento. Diagnóstico en Osteología 2000; 2: 4-6

39. Churchill EB. The operative treatment of hyper-parathyroidism. Ann Surg 1934; 100: 606-612.

40. Bauer W, Federman DD. Hyperparathyroidism epitomized: the case of Captain Charles E Martell. Metabolism 1962; 11: 21-29

41. Enersen OD. Who named it? http://www.whonamedit.com/search php?qs=Albright $+\&$ submit $=$ Search

42. Copp DH, Cameron EC, Cheney BA, Davidson AGF, Henze KG, 1962 Evidence for calcitonin - A new hormone from the parathyroid that lowers blood calcium. Endocrinology 70: 638-649.

43. Molina-Vélez I, Borrero J. Hiperparatiroidismo primario, presentación de dos casos. Antioquia Méd 1962; 12 (6): 335-356

44. Casas Morales R. Hiperparatiroidismo en Colombia. 1991, Bogotá, Instituto Nacional de Salud.

45. Ramírez J, Jácome A, Delgado C, Matuk A: Problemas diagnósticos del hiperparatiroidismo primario; presentación de cinco casos. Acta Med Col. 5:395 406, 1980

46. Jácome A, Matuk A, Gartner G, Ramírez J: Osteítis fibrosa quística - Manifestación común del hiperparatiroidismo primario. Acta Med Col 8:326329,1983

47. Jácome A, Reina R, Matuk A, Jiménez L. Localización de adenomas paratiroideas por medio de la tomografía computarizada. Acta Med Colomb 1988 13 (2): $95-97$

48. Ardila E, Mautalen C, Jiménez C. Osteoporosis en Iberoamérica. Editoria Manual Moderno (Bogotá-México), 2012. 585 páginas. 